RUNNING HEAD: Dynamic Risk Factors

\title{
Dynamic Risk Factors and Constructing Explanations of Offending: The Risk-Causality Method
}

\author{
Roxanne Heffernan \\ Victoria University of Wellington \\ Tony Ward \\ University of Ghent and Victoria University of Wellington \\ Stijn Vandevelde \\ Lore Van Damme \\ University of Ghent
}

Corresponding Author:

Dr. Tony Ward

School of Psychology

Victoria University of Wellington,

PO Box 600.

Wellington, New Zealand.

E-mail: Tony.Ward@vuw.ac.nz 


\begin{abstract}
The explanation of offending and reoffending currently relies upon the Risk-NeedResponsivity model's concept of “criminogenic need” (Andrews \& Bonta, 2010; Bonta \& Andrews, 2017) or dynamic risk factors (DRF). This is problematic because the DRF construct is predictive rather than explanatory, and suffers from a number of conceptual problems. Relatedly, the identification and management of DRF in individuals who have committed crimes is an overriding concern for the criminal justice system. It drives the formation of correctional policy and the funding of treatment programs in prisons, forensic hospitals, and probation services. Significant theoretical work is required in order to transform DRF into explanatory tools which are useful for research and practice. In this paper we outline problems with DRF which obstruct this process and then sketch out a possible way forward, the Risk-Causality Method, with its three phases of deconstruction, analysis, and reintegration.
\end{abstract}

Keywords: explanation of crime, dynamic risk factors, risk factors 


\section{Introduction}

The identification and management of dynamic risk factors (DRF) in individuals who have committed crimes is an overriding concern for the criminal justice system. It drives the formation of correctional policy and the funding of treatment programs in prisons, forensic hospitals, and probation services. Structured risk assessment has demonstrated marked success in predicting recidivism and provides a principled basis for allocating individuals to intervention streams (Andrews \& Bonta, 2010; Hanson \& Morton-Bourgon, 2009; Olver, Stockdale, Wormith, 2014). DRF appear to add incremental value in predictive algorithms and are increasingly being imported into research and treatment domains as explanatory constructs (Cording, Beggs-Christoferson, \& Grace, 2016). In their recent book Bonta and Andrews (2017) state that "dynamic predictors of criminal conduct or criminogenic need factors have great practical relevance because they inform interventions that reduce criminal behavior by identifying the targets of treatment” (p.20, italics in the original). This view strongly suggests that DRF play a causal role in reoffending, and therefore ought to be a focus of explanations of crime and taken into account when designing intervention programs.

The application of risk-related data to form explanations of individual behavior requires robust theoretical knowledge, whether explaining group level phenomena or individual offending in the form of forensic case formulations (Hart, Sturmey, Logan \& McMurran, 2011; Lewis \& Doyle, 2009; Logan \& Johnstone, 2013). Appealing to causes goes well beyond observing correlations between events, and frequently will refer to etiological (i.e., instigating causes) and compositional (i.e., processes that constitute or underlie the phenomena) mechanisms. These mechanisms are responsible for producing the affective, cognitive, biological, behavioral, and social phenomena associated with serious normative violations (i.e., crime), and by doing so, 
they are crucial in guiding treatment. Thus the identification of relevant mechanisms is an explanatory not a predictive task (Ward \& Fortune, 2016a).

A pressing concern is whether or not DRF can actually do the jobs prescribed for them by researchers and practitioners (Andrews \& Bonta, 2010; Bonta \& Andrews, 2017; Mann, Hanson, \& Thornton, 2010; Hart \& Logan, 2011). It has been suggested recently that DRF in their current form are best thought of as (at least partially) symptom-like features of individuals and their environments which are generated by causal mechanisms rather than being causes themselves - they are a good starting point to think about the causes of offending (Ward \& Fortune, 2016a, 2016b; Ward \& Beech, 2015). A difficulty with recent work on incorporating DRF into explanations of (re)offending, risk assessment, and/or forensic case formulations is that it is assumed that they are coherent constructs (e.g., Hart, Sturmey, Logan \& McMurran, 2011; Sturmey \& McMurran, 2011). In our view this is not the case, and DRF are better conceptualized as red flags (i.e., symptom-like features) that indicate the existence of problems but cannot explain why they have arisen or persisted. They may be useful predictors but are not coherent explanatory constructs. The current emphasis on empirically established lists of DRF or criminogenic needs embedded within a theory of criminal conduct (Andrews \& Bonta, 2010; Bonta \& Andrews, 2017) in both research and practice falls short of causal explanation (see Ward \& Fortune, 2016a).

The major goal of this paper is to explore ways in which DRF can be used to explain offending behavior and to guide assessment and treatment. We offer one possible method for utilizing DRF within explanations in both research and clinical work: the Risk-Causality Method (RCM). We first argue for the value of adopting a methodological framework within which to conduct research. Second, the problems with the current conceptualization of DRF are noted, drawing from an example of the general dynamic risk category of intimacy deficits. Third, we follow this with a 
discussion of possible solutions from the recent theoretical literature, and comment on their potential and limitations in addressing the problems currently faced by DRF. Fourth, we present the RCM in detail and apply it to the example of emotional congruence with children. Fifth, practice implications of the RCM are briefly discussed. Finally, we conclude with some remarks concerning its potential contribution to the field.

It is hoped that the arguments and suggestions developed here will encourage future research into the composition of DRF and their role in informing more useful explanations of offending and related phenomena. What we are offering in this paper is a methodological framework to help researchers and practitioners infer the possible causes of crime and its related problems. The use of the RCM can make the task of translating DRF into specific causal processes easier, and therefore bridge the current theoretical gap between prediction and explanation in both research and practice domains.

\section{Methodological Frameworks in Research}

Scientists employ specific methods to detect empirical phenomena, and construct models or theories of the causal mechanisms thought to be responsible for their occurrence. A general methodological framework offers a way of unifying the diverse range of cognitive tasks involved in reaching these descriptive and explanatory goals. Following Haig (2014), science typically proceeds as follows: constrained by a developing problem comprising a set of empirical, conceptual, and methodological considerations, certain data are brought to the researcher's attention and are ordered via the detection of one or more phenomena. Once detected, these phenomena are explained by abductively inferring the existence of an underlying causal mechanism. Here, abductive inference involves reasoning from a presumed effect (i.e., the 
phenomenon) to its explanation in terms of an underlying causal mechanism (i.e., the theory). From an initial judgement of the plausibility of such an explanatory theory, attempts are made to elaborate on the nature of that mechanism, frequently by way of constructing plausible models. When the theory is well developed, it is evaluated on a number of dimensions including its empirical adequacy and criteria principally to do with the explanatory worth of the theory.

The value of adopting a theory of scientific method is that it unifies the diverse tasks that constitute research within an overarching general framework: formulating an initial question, designing a study, choosing methods for collecting data, analyzing data, detecting explanatory targets, inferring etiological and compositional factors (i.e., the structures and processes constituting mechanisms that underlie phenomena), and so on. Without a methodological framework it is easy to get lost in the research process and run the risk of squandering limited cognitive, social, and financial resources. Consistent with this view, it makes sense to construct general methods of inquiry to guide researchers in their attempts to identity and explain crime and its related phenomena. If we accept that DRF track causal processes in some way (see below), then coming up with a general method to help isolate and model potential causes at multiple levels of analysis will be invaluable. This kind of methodological framework will be nested within the type of general scientific method discussed above; it should provide assistance in picking out promising causal factors from the research and clinical literature, and in constructing rich descriptive models of their constituents and subsequent causal impact. The Risk Causality Model (RCM) is an example of such a methodological framework.

\section{Dynamic Risk Factors}


Dynamic risk factors (DRF) are changeable features of individuals and their environments which predict higher rates of reoffending. They are also referred to as "criminogenic needs" and there appears to be consensus that they are at least potential causes of criminal behavior (Bonta \& Andrews, 2017; Mann et al., 2010). DRF are contrasted with static risk factors, which are risk correlates such as criminal history, age, and gender unable to be changed via intervention. Because of these defining features, DRF are used as both predictors of risk and targets for change. This is reflected in the evolution of assessment tools from containing primarily static markers of risk to incorporating dynamic variables which can be used to guide practice. The recruitment of DRF to explain crime, formulate cases, and inform treatment is now standard practice. Researchers propose that the most strongly supported variables should be emphasized in both assessment and treatment of those who have committed offences (Andrews \& Bonta, 2010; Bonta \& Andrews, 2017). For example, in the case of individuals who have committed sexual offences, emotion regulation problems, deviant sexual interests, offence supportive attitudes and beliefs, and social intimacy deficits have all been identified as DRF in the literature (Mann et al., 2010).

While it is assumed that DRF have an explanatory role in research and practice, there are a number of conceptual problems with these predictive constructs which we will briefly outline here. (1) The first problem is that DRF lack coherence, they are composite constructs which contain a number of different types of variable (Ward \& Fortune, 2016a). While they contain causal strands, in their standard form they are more like general categories that also incorporate contextual (e.g., gang membership), behavioral (e.g., watching child pornography), and psychological state aspects (e.g., feeling lonely). (2) Due to the co-existence of these composite categories and their multiple potential causal strands, DRF lack specificity. That is to say they are unable to identify which potential cause is relevant for explaining certain phenomena. (3) 
DRF lack precision and suffer from the grain problem, which means that there is little agreement concerning which level of abstraction is the appropriate one to interpret them at. They are often formulated at various levels: as general or umbrella categories or as more fine grained categories composed of specific features. (4) DRF lack factualness because they are not scientific kinds. They are normative constructs which only exist due to their co-occurrence with behaviors and outcomes which society has deemed harmful and/or unlawful.

In order to make these conceptual problems more concrete, we will apply them to the example of lack of emotionally intimate relationships with adults or intimacy deficits. This DRF category has strong empirical support for its association with sexual recidivism, and relies upon contextual evidence such as having no stable partner relationship currently or in the past, or having intimate relationships characterized by conflict and infidelity (Mann et al., 2010). It encompasses those who desire and those who avoid intimacy, and it has been acknowledged that "these varying facets of dysfunctional intimacy may have different underlying pathologies and so may lead to different treatment targets" (Mann et al., 2010, p. 201). It is further suggested that intimacy deficits could be an indicator of other DRF, such as sexual deviance, attachment problems, and poor emotion-management (Mann et al., 2010). Thus it is unclear what exactly the term "intimacy deficits" refers to, and how it might increase risk at the individual level (i.e., it lacks specificity- problem 2, see above). In addition, it is a normative category (problem 4, see above) in the sense that intimacy levels vary across the population, and are only deemed to be at a dysfunctional level when they co-occur with more serious norm violations such as sexual offending.

In order to illustrate the remaining two problems, lack of coherence (problem 1, see above) and lack of precision (problem 3, see above) we will explore the composite nature of the category of "intimacy deficits". The cluster of factors which 
hang together across explanatory levels and grains of analysis (i.e., from general categories to specific processes) to cause or constitute this DRF include (but are not limited to):

- Cultural/contextual level: Norms specifying the types of relationships appropriate and what these should involve; gender norms; ideal sources and amount of intimacy (e.g., intimacy deficits); laws (e.g., age of consent), and social opportunities for connection.

- Interpersonal/social level: Interpersonal skills (e.g., communication); social learning; support/advice, social roles; expectations, and responsibilities.

- Phenomenological/psychological level: emotional connection/congruence; empathy; sexual preferences; beliefs about relationships (i.e., self and others); perspective-taking; emotion-management; and attachment style.

- Neuropsychological level: brain regions and neurotransmitters, such as oxytocin, vasopressin, pre-frontal cortex, and hormones that underpin psychological problems and experiences indicative of intimacy problems.

- Biological level: biological sex; sexual arousal; physical health; and physical attributes (i.e., size, attractiveness).

Each level of description relies upon various sources of evidence, and varies in its level of abstraction. The umbrella category "intimacy deficits" encompasses more specific features at lower levels, such as emotional (e.g., congruence with children) and cognitive (e.g., beliefs about children and sex) processes. No level on its own can provide an adequate explanation of intimacy deficits, as unique properties emerge across levels. These examples are by no means exhaustive, but hopefully illustrate the range of influences evident in just one DRF category, and also the significance of 
overlap between different categories (e.g., intimacy, cognition, emotion, and sexual). In addition, the inclusion of different types of constructs within a single DRF category is incoherent and creates confusion. This discussion provides support for our assertion that DRF categories are of little use for the purposes of explanation. They are acceptable predictors, but if they are to explain (re)offending or be imported into the treatment domain (via case formulation) they need to be reworked (see Ward \& Fortune, 2016a for an in depth discussion of the problems of coherence, specificity, precision/grain, and normativity).

In order to outline the scope of DRF categories, we provide a summary of the core DRF domains for sexual offending against children, and the more specific factors within each category. The DRF contained within Table 1 below are taken from Mann et al.'s (2010) list of the most well supported and promising psychologically meaningful risk factors, meaning that they reliably correlate with reoffending and are plausible causal. In addition to providing useful starting points for inquiry, Table 1 further illustrates the composite nature of DRF, and links these with the sorts of practices within which they manifest, and examples of causal processes which may be relevant for each.

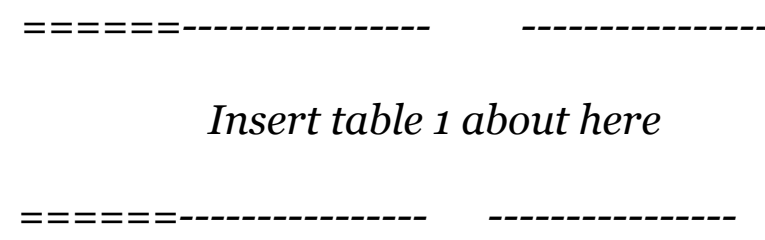


We will now discuss several overlapping suggestions from the literature concerning how to utilize DRF in research and clinical domains, and then outline the Risk-Causality Method.

\section{Incorporating DRF into Forensic Explanation}

There are a number of suggestions in the existing literature for how to best utilize DRF in the explanation of (re)offending and treatment planning. We will consider five of these here: (1) ignore the above problems and treat DRF as causes; (2) evaluate them against a set of risk factor causal criteria; (3) utilize a risk-matrix; (4) reconceptualize DRF as (proxies of) impairments in agency; and (5) locate DRF within social exemplars/practices. Our conclusion is that there is potential value in combining these models within a broader methodological framework (the RCM). The models and theoretical ideas we discuss in this section have appeared only relatively recently in academic journals and books. In part this is because the conceptual status of DRF has not previously been questioned and it was simply assumed that some of them (at least) were causal constructs.

\section{DRF as Causes}

The first strategy involves accepting DRF in their current form, and relying on these as promising candidates for the explanation of (re)offending - essentially business as usual. However, due to the difficulties outlined above we do not think that this is a viable theoretical strategy, and suggest that it will eventually lead the field of forensic and correctional psychology into a theoretical dead end (Ward \& Beech, 2015). Reflecting on Bonta and Andrews (2017) depiction of the causal relationships between seven of their "big eight" risk factors and crime Heffernan and Ward (2017) observe: 
Rather than being an explanation of criminal behavior, the seven factors provide descriptions of problems typically observed in individuals who persistently offend and in their environments. At this point in time they are best viewed as broad areas indicating vulnerability rather than as specific causes of offending..... While this model describes the functional relationships between DRF and criminal conduct, in its current form it is unable to explain the onset and/or reoccurrence of crime...(p. 130).

\section{From DRF to Causal Status: Bradford Hill's Criteria}

One strategy which rejects the assumption that DRF are causes in their typical composite form, asks researchers to evaluate the potential causal elements of DRF against standards such as Bradford-Hill's (1965) criteria for causal inference in the medical epidemiology field. In essence, Bradford-Hill's aim is to provide a set of criteria to elevate risk factors from the status of predictors to that of causes. He argues that it is reasonable to infer that a risk factor is a cause of a disease/problem if it is consistent with the following guidelines:

1) Strong statistical association with a specific outcome

2) Consistency (e.g., place, circumstances, time, and observers)

3) Specificity (e.g., to particular groups, body systems and sites, and diseases)

4) Temporality (a putative cause precedes an outcome)

5) Biological gradient (e.g., expect a decrease in effect with a decrease in cause)

6) Plausibility (i.e., is the cause reasonable within the context of current knowledge? Ideally an etiological/causal mechanism should be identified)

7) Coherence (i.e., does it cohere with knowledge about the domain? Established facts act as epistemic constraints on causal inference) 
8) Experimental manipulation (i.e., evidence from well-designed studies supports a cause and effect relationship)

9) Analogy (i.e., the existence of other similar causal relationships)

DRF in their current form arguably satisfy a number of these causal status criteria, however they fail to meet others. On the plus side of the equation: they are empirically derived from risk correlates (1); appear to hold across offending groups and raters (2); and have been observed to precede reoffending in longitudinal studies (4). However, on the negative side: they are not problems or outcomes which are specific to offending populations (3); they do not reliably exhibit the expected increases and decreases in conjunction with recidivism rates ( $5 \& 8)$; they are not theoretically coherent and do not refer to causal mechanisms (6 \& 7); and analogous concepts have not demonstrated causal relationships ( 9 - see for example the Research Domain Criteria project which aims to identify causal processes underlying psychopathology; Lilienfeld, 2014). The problem is that unlike the questions of causality posed by epidemiology (e.g., the relationship between the risk factor of smoking and lung cancer), risk factors do not refer to causes in any direct sense, and are inherently vague. By "inherently vague" we mean that in their current composite form they could refer to any number of possible situations and properties. Without further conceptual and theoretical analysis they simply contain too many causal possibilities to be confident of their role in facilitating norm-violations. In addition, many of the elements of causality contained within DRF categories have not yet been researched extensively with offending populations, and so it will be difficult to ascertain whether they meet the above criteria.

The Bradford-Hill criteria outlined above offer useful suggestions for ascertaining whether or not specific factors summarized by DRF can be justifiably 
considered by researchers and practitioners as possible causes or not. However, because these guidelines do not directly assist in the identification of the mechanisms underlying DRF, in the criminal justice area they are best utilized as initial filters to help identify possible causes of crime and its problems.

\section{Dynamic Risk Research Framework: Risk Matrix}

A third suggestion for the investigation of DRF was put forward by Ward and Fortune (2016b): The Dynamic Risk Research Framework (DRRF). This approach involves identifying the causal elements of DRF by referring to the psychological processes inferred in recent psychopathology research (i.e., it is heavily based on the Research Domain Criteria or RDoC project matrix; Lilienfeld, 2014). Briefly, the aim of the RDoC project is to develop new ways of classifying mental disorders based initially on five domains of psychological processes and their instantiation in neurobiology. The DRRF proposes that potential causal processes should be teased apart within a matrix spanning multiple levels of analysis. The Y axis contains six categories of possible causal processes: negative affective systems, positive affective systems, cognitive systems, self-regulation systems, intrapersonal social systems and interpersonal social systems (Ward \& Fortune, 2016b, divided the RDoC domain of social processes into two separate categories). The $\mathrm{X}$ axis contains four different levels of analysis for each of the putative causal processes: biological, behavioral, phenomenological, and contextual (reduced from the RDoC's six units of analysis). The purpose of multi-level data collection is to provide various types of evidence for causal processes, which can then be used to form more comprehensive explanations of risk-related phenomena (i.e., add explanatory depth). For example, emotional congruence with children contains a number of possible causal processes including a fear/anxiety response to adults, which can be investigated in terms of neural networks and physiological processes, thoughts, beliefs, and emotions, triggering contexts, and 
resulting behaviors. A virtue of filtering DRF through the DRRF matrix with its core psychological domains and levels of analysis is that their various components and relationships with each other can be more easily discerned. This example will be analyzed in greater depth when we incorporate the DRRF into the Risk-Causality Method.

The DRRF advocates for an understanding of normal or adaptive functioning, in order to better understand dysfunctional processes. The problem is that crime is not necessarily linked with dysfunction; mechanisms could be functioning as intended but directed towards maladaptive or harmful goals (i.e., the normative component). Nevertheless, it is likely that impairments will be present and relevant for a number of individuals, and that explanations pitched at the behavioral and relational (or possibly contextual) levels of analysis can capture the normative components of DRF, while the biological and phenomenological levels deal more directly with facts about the integrity of these systems. Therefore, we suggest that this approach has utility in guiding the theoretical exploration of DRF once they have been broken down into their causal, contextual, and symptom-like (behaviors and mental states) variables. Agency Impairments

The fourth approach to the reconceptualization of DRF rests upon the concept of agency, the capacity for and process of goal-directed behavior. Recent theoretical papers attempting to link DRF with offending have highlighted the importance of agency and the associated view of offending as goal-directed behavior (Heffernan \& Ward, 2015; Heffernan \& Ward, 2017; Serin, Chadwick \& Lloyd, 2016; Thornton, 2016). Stressing the importance of focusing on actions as well as underlying mechanisms, Ward, Wilshire, and Jackson (2018, p. 199) comment "in the context of forensic psychology, our primary goal is to generate etiological explanations of 
behavior. Since the targets of our explanations are complex behaviors, the psychological level of description has a privileged status here".

From this perspective, DRF are broad categories referring to (i.e., are proxies for) impairments in the capacities underlying agency (e.g., emotion, beliefs, desires, planning, counterfactual thinking, expectations, etc.) and/or social circumstances (e.g., gangs, poverty, unemployment) that cause behavior that is harmful and/or illegal in particular contexts. This means that DRF are contextually bound; what may be a strength or weakness for one individual or context may not be for another. While different theories infer diverse psychological structures and processes, the assertion is that DRF should be conceptualized as problems with the components of agency (intentional, goal directed behavior) and/or the contexts in which it is exercised. For example, intimacy deficits could be partially caused by fear responses to adults due to impaired theory of mind capacities (i.e., "women are cruel"), and poor problemsolving skills could be due to impairments in generating multiple options for action or difficulties with counterfactual thinking.

Conceptualizing DRF as problems with the capacities underpinning agency can begin to overcome the issue of their composite nature, as aspects of various DRF are dispersed throughout the agency process. To take the example of emotional congruence with children: an individual values and is motivated to achieve intimacy (i.e., relatedness and pleasure) but believes that adults will harm him; he is in a situation where he feels threatened and lacks the necessary skills to regulate these feelings and he is in an environment with vulnerable children; these impairments and situational factors interact to cause an offence. The focus on human agency means that the first-person, intentional level of explanation is prioritized. Because explanations tend to focus primarily on behavior and the psychological and situational explanatory levels they do not necessarily incorporate social, cultural, or biological aspects well 
and thus need to be supplemented with explanations spanning these additional levels of analysis. They are descriptions of action sequences and as such useful for (1) identifying salient patterns to analyze further, and (2) informing the critical explanatory targetsof theories of crime.

\section{Exemplars and Social Practices}

The final way of analyzing DRF to be discussed here is that of exemplars and normative social practices. The basic idea is to embed the description of DRF within their relevant temporal and social contexts. The difference between agency models and the exemplar/practices approach is that the former is focused on what persons do while the latter is on problems and their manifestation.

Firstly, "an exemplar is a representation of the typical course and symptoms of a mental illness, whereas a model is a representation of those symptoms, that course, and the causal determinants of both of them. A model is an exemplar together with an explanation" (Murphy, 2006, p. 206). In the forensic/correctional context, exemplars could be prototypical offence action sequences, such as carefully planned "grooming" behaviors and the sexual abuse of children. Practices are coordinated sets of actions centered upon certain goals and their associated norms (Ward \& Heffernan, 2017). Norms are evaluative in nature and spell out whether or not an activity is done properly; whether it meets the socially accepted relevant standards. Practices typically depict normative behavior, and DRF represent violations of these norms. For example, in the case of normative sexual behavior: partners should be cognitively competent adults, sex should occur in private settings, and ought to only involve sexual behaviors that are agreed to and are relatively harmless. In the case of intimate romantic relationships: they should only occur between consenting adults, ought to be reciprocal, should include personal disclosure of fears and needs, ought to incorporate 
caregiving and sexual components, should contain shared activities and responsibilities, and so on.

The practices and exemplars approach provides a useful way to identify the relevant norms and social/cultural models which govern human behavior, including norm-violating patterns of behavior (i.e., illegal practices), which rely upon the functioning of agency capacities. It respects the first person perspective in providing unique insight into intentional practices, but also makes room for a third person (i.e., an observer) perspective in the form of norms and social expectations. Thus it deals with both the normative aspects of DRF, as well as assuming that the external conditions for agency are present. However, this approach to reworking DRF lacks depth concerning the structures and processes underpinning agency. The intentional level of explanation cannot tap into the range of sub personal mechanisms which underlie goal-directed practices. Like the agency approach above, this needs to be supplemented with a more in-depth exploration of causal processes across additional levels of explanation.

\section{The Risk-Causality Method}

Each of the above potential ways of reworking DRF to causally explain normviolations and to guide treatment has its own list of strengths and weaknesses. While they propose that the deconstruction and investigation of possible causal elements should play an important role in directing research into the causes of norm-violations, they fail to provide sufficient guidance to researchers and practitioners. Capitalizing on the strengths of the above models we have developed the Risk-Causality Method (RCM) conceptual framework, which structures the analysis of DRF into three phases: deconstruction, analysis, and reintegration. In the RCM, each of the above suggestions for transforming DRF into possible causal elements plays a valuable role, 
albeit in different phases of the model. The specific theories and models used in this section should be viewed only as examples to illustrate the utility of the RCM and ought not to be regarded as the correct way to unpack each phase. What is unique is the RCM methodological framework, not the specific models and ideas associated with each of its three phases. Future use of the model needs to draw from a greater variety of theories supported by multi-disciplinary research. We will now outline the three phases of the RCM in general terms before applying it to an example of a DRF.

RCM Phase One: Deconstruction

The first phase of the RCM logically follows previous research which regards DRF as useful markers of (or as red flags for) crime related factors. Their role in the inquiry process is to direct attention to potential causes, relevant contextual features, and salient behavioral and mental state variables ("symptoms"). It is important during this first step of deconstructing DRF to view them within the context of goaldirected practices, and to consider whether they could potentially meet Bradford-Hill's (1965) causal criteria. This will ensure that both the factual and normative aspects of DRF are considered. For example, Mann et al.'s (2010) list of empirically support DRF for sexual offending are a useful starting point. While they do not meet all of BradfordHill's (1965) criteria, they are currently the closest thing we have to psychologically meaningful constructs (i.e., agency capacities) which are linked with this particular type of norm-violation (Mann et al., 2010).

Once a candidate DRF is identified, researchers and practitioners should generate an exemplar (i.e., a typical description) of problems within this category; these anchor the first phase. Researchers can then describe the behavioral or mental state (i.e., self-report) symptom-like aspects of the DRF, the contexts in which these are observed, and the range of potential causal processes underpinning these. The identification of the different types of constructs contained within each DRF makes it 
easier to think about the causes of crime and the way they interact with contextual variables and each other.

In order to provide structure to the identification of these causal processes, researchers should consider the six types of causal processes listed in the DRRF in the section above: negative affective systems, positive affective systems, cognitive systems, interpersonal social systems, self-regulatory systems, and intrapersonal social systems. Carefully filtering the types of causal processes referred to by DRF will help researchers constrain their subsequent analyses of the relevant mechanisms.

RCM Phase two: Analysis

The second phase of the RCM begins with the list of promising causal candidates from phase one. In the analysis phase researchers should refer each of the possible causes to something like the DRRF matrix to discern their possible specific causal components and the evidence for them at different levels of analysis. This process draws from psychological, social, and neuroscientific theories to infer possible mechanisms (causes) and processes. While this is potentially the most complex phase, it is a very important one, and requires thorough investigation in order to provide comprehensive multi-level accounts of the phenomena in question (i.e., those identified in phase one).

The result of the second phase will be models of each potential causal process (repeated for all of the DRF). These models should spell out how a system ought to function, and thus be able to identify whether or not it is operating as it should. In addition, these models will require reintegration with a more general conception of human agency (see the next phase) in order to understand their interaction and influences on behavior - no system on its own can explain how these processes contribute to serious norm violations.

RCM Phase Three: Reintegration 
In the third phase of the RCM the goal is to knit the information provided in the earlier two phases together. The various models and relevant contextual features, mental state and behavioral variables are integrated within a model of goal-directed practices, such as the Predictive Agency Model (PAM; Heffernan \& Ward, 2017). This step illustrates the role of the causal processes in problematic engagement in normative practices, such as intimacy, sex, coping, and so on. In this sense the final step is reintegrating information concerning the various problems or impairments (the causal processes) underpinning norm violations, with the sorts of practices and environments in which they manifest. The depiction of the RCM so far has been very abstract, so we provide a visual model below, and in the following section we apply the RCM to a concrete example.

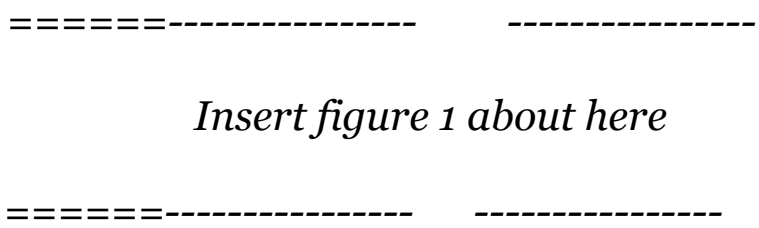

\section{Illustrating the Risk-Causality Method: Emotional Congruence}

In this section we outline the application of the three phases outlined above to the ongoing example of emotional congruence with children. This will illustrate the use of the RCM in guiding theoretical research into the mechanisms underlying DRF. We make a distinction between the theoretical task of developing general explanations of DRF and the more specific task of individual case formulation. This paper is primarily focused on the first task but has implications for the second. Once the initial theoretical work has been completed in line with the RCM, simplified versions of the models constructed can be utilized in practice with individual cases if and when they are deemed relevant. 
RCM Phase One: Deconstruction

Our example DRF, emotional congruence with children is one of Mann et al.'s (2010) psychologically meaningful risk factors; thus it is reliably linked with sexual reoffending and is a plausible causal factor. It involves an individual experiencing intimate relationships with children as more emotionally satisfying than relationships with peers. This individual could find children easier to relate to and/or still feel like a child himself, and believe that children understand and accept him more than other adults. This often results in feeling like he is "in love" with his young victims, and to think of the relationship as reciprocal and mutually beneficial (Mann et al., 2010). An exemplar may read: He is unable to engage in the normal social practices of adult romantic relationship establishment, maintenance, and repair. This is because he values sex and intimacy with children positively and devalues or fears that with adults. He feels safe and valued around children, and believes that his victim was a willing participant in the relationship.

From here it is possible to deconstruct emotional congruence with children into different types of causal processes and contexts/situations that interact to generate the symptoms (i.e., behaviors and mental states) evident in individuals who feel emotionally drawn to children:

- Mental state/behavioral variables (i.e., symptoms): For example, feelings of fear, loneliness, desire, safety, excitement, hopefulness, despair, avoids intimacy with adults, makes statements reflecting beliefs about children as capable of consenting to and enjoying sex (i.e., willing participant), reports being in love with victims, uses "grooming" strategies to establish trust. 
- Contexts/situations: For example, has friendships and intimate contact with children, social isolation, lack of intimacy with adults, regular unsupervised access to children, deviant social networks.

- Possible causal processes:

- Negative affective systems: For example, fear and anxiety (i.e., fearful/avoidant attachment); loneliness; guilt/shame (i.e., post-hoc rationalizations).

- Positive affective systems: For example, views children as sources of reward and more likely to signal opportunities for love, sexual pleasure, and care.

- Cognitive systems: For example, displays attentional bias towards signs of affection from children or indicators that it might be possible to become involved with them sexually and emotionally (e.g., cues signifying vulnerability, lack of supervision); beliefs/schema support associations between children and sex (i.e., it is not harmful), developmental deficits (i.e., cognitive impairments).

- Intrapersonal social systems: For example, a tendency to view himself as vulnerable and unsafe, living in a dangerous world; lacks understanding of his motives due to expectancy and interpretational biases.

- Self-regulation systems: For example, lacks the capacity to soothe himself and effectively control negative physiological arousal; seeks interaction with children to do this and constructs elaborate grooming strategies to accomplish this based on problematic beliefs and goals.

- Interpersonal social systems: For example, theory of mind impairments (i.e., inability to take the perspective of and represent others' mental 
states), internal working models in which affiliation seeking strategies are entirely directed towards daily interactions with children, including sexual contact. These may be strengthened via involvement in deviant social networks that approve of sex and intimacy with children.

Thus, in this first phase we view DRF as instigators of inquiry (markers of causal processes), rather than as endpoints of inquiry. The causal processes identified during phase one are the targets for analysis in phase two, and are then reintegrated within a model of human agency in phase three.

RCM Phase Two: Analysis

The second phase involves an in-depth analysis of the potential causal processes identified, and use of the DRRF (Ward \& Fortune, 2016b) in order to investigate each of these systems across multiple levels of analysis: biological, behavioral, phenomenological, and contextual. Due to limited space, we will focus on the example of a negative affective system identified in phase one above: the tendency to view adults as threats and a source of fear and anxiety, resulting in avoidance of intimacy with adults and a preference for intimacy with children. At the biological level, this system could involve the amygdala, central nervous system, and associated physical responses to fear and anxiety (e.g., heart racing, dry mouth, and perspiration). At the behavioral level this system is primarily concerned with fight or flight responses, for example avoidance of interactions with adults or hostility and mistrust towards them. These behaviors could manifest in a lifestyle (i.e., social and physical contexts) characterized by "social isolation" or "intimacy deficits", and the seeking out children as a safer alternative. The contexts in which these systems are activated may include social events where unknown adults are present, rejection from adults, and where norms require social interaction. At the phenomenological level this 
system involves beliefs about adults (e.g., rejecting, dangerous, manipulating), memory and attentional biases (i.e., towards events that confirm these beliefs), and associated emotions such as fear, anxiety, and loneliness.

In order to gain a deeper understanding of how things have gone wrong, researchers need to explain how a threat detection system ought to work. Its functioning likely exists upon a continuum, with a healthy range existing within the middle, and sub-optimum functioning when the system is over or under-functioning. It is also context-dependent and normatively defined, for example it is normal and adaptive to be more sensitive to interpersonal threat in prison, whereas intimacy practices require a certain level of trust and vulnerability. For this reason, the contextual level must be explored and integrated with lower levels of analysis. The analysis phase involves integrating evidence from a number of disciplines, for example psychopathology (e.g., social phobia), evolutionary psychology (e.g., the selective advantage of fear responses), developmental psychology (e.g., attachment), and neuropsychology and biology will be particularly useful for lower level explanations. This work remains to be done, and is outside the scope of this paper. It is anticipated that the output from phase two will consist of a number of specific models centered upon each identified causal process, and spanning multiple levels of analysis.

It is also worth noting at this point that the functioning of the negative affective system (and others) is relevant to a wider range of symptoms and behaviors which reflect the violation of accepted social norms, not just emotional congruence with children and sexual offending. For example, dysfunctional threat detection may play a role in social anxiety, general relationship problems, violent offending, and substance abuse. The same underlying mechanisms can produce different behaviors (both legal and illegal) depending upon the context and the functioning of other systems. It may lead to sexual offending against children when paired with a sexual preference for 
children, beliefs about the ability of children to consent to sexual intimacy, or selfregulation problems (i.e., intoxication lowering inhibitions) in certain contexts.

RCM Phase Three: Reintegration

The third phase involves the reintegration of causal processes and their associated systems (i.e., local theories) within an agency framework containing emotional capacities; psychological representations; cognitive processes; formulating goals and plans; implementing plans; evaluating outcomes; and modifying plans and goals within day to day activities (i.e., goal-directed practices). The goal of this phase is to provide an understanding of how the putative causal processes contained within each DRF interact with each other and contextual features to produce offending behavior. For illustrative purposes, we will embed our example within the previously developed Predictive Agency Model (PAM, Heffernan \& Ward, 2017).

In our (speculative) example, underlying fear/anxiety towards adults is an internal working model of the self as vulnerable, other adults as dangerous and children as safe and accepting. In terms of general affect, the individual is lonely due to avoidance of peers, and is overly sensitive to interpersonal threat. In social situations, such as family gatherings, he tends to pay attention to social cues which support his internal working models, and construct situation-specific models which are in line with these. For example, if certain adults do not talk to him it is perceived that they dislike and want to harm him. He has developed the ability to quickly create friendships with children, and is good at putting children at ease and getting parents to trust him (i.e., he can anticipate their reactions to his interest in children). He has excellent planning abilities and is always thinking several moves ahead; he rarely acts impulsively. 


\section{Summary}

We have taken just one example of a DRF and used the Risk-Causality Method to tease out possible causes, contexts, and behavioral and mental state variables ("symptoms") in order to explain how they might manifest in harmful social behavior. It is clear from this example that emotional congruence with children is linked to other DRF, such as problematic beliefs and deviant sexual preferences. An advantage of breaking down DRF and embedding them in behavior in this way is that it becomes easier to formulate cases. Once further theoretical work has been completed, practitioners will have access to a causal model not simply a statistical/predictive one. This offers greater potential for accounting for diverse behavioral features with an integrated set of mechanisms, is more individualized, and has greater explanatory depth - it provides more information than stating that "this individual emotionally identifies with children". In addition, because of its focus on mechanisms and their ability to function within certain contexts, the RCM has the potential to explain the role of protective factors in desistence, another area that has been theoretically neglected.

\section{Implications for Practice}

Case formulation is the analogue of theory construction in the practice domain: the creation of an explanatory model that accounts for the onset, interrelationships, and maintenance of problems associated with crime (Hart \& Logan, 2011; Sturmey \& 
McMurran, 2011; Ward \& Beech, 2015). Forensic and correctional case formulation is used to guide the rehabilitation of those who have committed offences, and until recently has received relatively little attention in the literature (Sturmey \& McMurran, 2011). It is an important task, which involves the gathering of risk-related data and construction of a plausible explanation (i.e., a hypothesis) for how and why these factors cause and maintain offending. Typically, it is based upon the widely accepted "propensity model" of risk, where a number of long term vulnerabilities, known as Dynamic Risk Factors (DRF), interact with environmental triggers and opportunities to influence behavior (Beech \& Ward, 2004). Case formulation bridges the gap between prediction and explanation. It guides practice via the integration of empirical knowledge and theoretical understanding of DRF. The problem facing forensic and correctional practitioners at the moment is that existing case formulation models assume that DRF are theoretically coherent constructs, when in our view, they are not (see Sturmey \& McMurran, 2011).

Our primary goal in this paper has been to consider the relevance and value of the RCM for researchers rather than practitioners. This is primarily because it makes little sense to construct case formulation and intervention plans without a reasonable understanding of what the causes of crime related phenomena are. Simply relying on DRF as treatment targets is a mistake as they do not reliably identify underlying causes at all; they are in effect summary labels for possible causes, contextual features, behavior, and mental state variables. Strictly speaking, DRF do not exist for the purposes of treatment, there is little point targeting symptom-like summaries and assuming that this will alter the causal mechanisms generating them. However, from a pragmatic viewpoint the RCM can play a valuable role in structuring clinical inquiry and, in conjunction with knowledge of etiological theories, risk assessment, and classification models, can assist practitioners to arrive at a working explanation of an 
individual's crime related problems. It can bridge the gap between risk assessment and intervention, and ensure that practitioners carefully consider the explanatory possibilities offered by DRF and avoid the trap of assuming they directly pick out causal factors. An advantage of structuring assessment and subsequent treatment by the RCM is that it confers a degree of epistemic skepticism on practitioners' conceptualizations of clients and reminds them that they critically depend on theoretical and methodological assumptions.

\section{Conclusions}

We have argued that DRF should not be accepted at face value as possible causes of offending because of the problems of incoherence, lack of specificity, the grain problem, and their marked normative status. We introduced the RCM with its phases of deconstruction, analysis, and reintegration as a promising way forward in the investigation of the potential causal elements contained within the broad DRF categories. We propose that the RCM can be usefully applied to DRF to "boot strap" theory development and eventually provide a valuable source of information for formulating cases, when paired with a case-formulation model such as the Abductive Theory of Method (ATOM; Haig, 2012; Ward, Clack \& Haig, 2016). At this stage it is intended primarily as a research model which aims to provide a link between theory and practice.

We have concentrated in this paper on the role of DRF in explaining offending and its associated problems. The reason for this is that there is strong theoretical justification and relevant evidence that these risk predictors may also be causes of (re)offending. However, the RCM can also be utilized with other types of potential causal factors such as those in etiological theories or implicit within classification systems. For example, attachment models of intimacy deficits in sexual offenders 
propose that problematic social behavior is in part caused by impaired internal working models of relationships (Smallbone \& Dadds, 2000). Maladaptive core beliefs of the self and other people are aligned with interpersonal strategies designed to protect individuals from rejection and overwhelming negative emotional states. Alternatively, according to the self-regulation model of the offence process, individuals who commit sexual offences vary in terms of their core values, goals, and strategies for managing problematic desires and situations. These differences manifest in distinct patterns of offending behavior (Yates \& Kingston, 2006). In both of these examples, the RCM could be applied to possible casual mechanisms such as impaired internal working models or self-regulation styles to develop richer theoretical depictions of them. In addition, it would help researchers to trace their links to various mental states, behaviors, and contextual features, and to spell out any possible treatment implications. Thus, the RCM has the potential to unify theory development tasks in a variety of research domains, and has a much wider reach that simply breaking down DRF.

Finally, even at this preliminary point of the RCM's development, practitioners may find it useful to guide the assessment and treatment of individuals who have committed offences. Simply relying on existing case formulation models or etiological theories that assume that DRF (as currently stated in the literature) are possible causes is likely to result in overly general, poorly integrated formulations (i.e., everyone looks the same or possible hypotheses are overlooked). Our suggestion is that in the domain of intervention DRF should only be used to indicate general problem areas and regarded as summaries of possible causes, contextual factors, behavioral, and mental state variables. By the processes of deconstruction, analysis, and reintegration we can put DRF to work in ways that are likely to give us a deeper understanding of why and how individuals act in ways that harm other people. The RCM enables researchers and 
practitioners to bridge the gap between theory and intervention in the criminal justice system. In order to intervene to effectively reduce crime it is necessary to move beyond risk assessment and management. Ultimately this requires us to appreciate how risk factors exert causal effects and how best to ameliorate their influence. 


\section{References}

Andrews, D. A., \& Bonta, J. (2010). The psychology of criminal conduct. New Providence, NJ: Anderson Publishing.

Bonta, J., \& Andrews, D.A., (2017). The psychology of criminal conduct (6th ed.). New York, NY: Routledge.

Bradford Hill, A. (1965). The environment of disease: Association or causation?

Proceedings of the Royal Society of Medicine, 58, 295-300.

Cording, J. R., Beggs Christofferson, S. M., \& Grace, R. C. (2016). Challenges for the theory and application of dynamic risk factors. Psychology, Crime \& Law, 22(1-2), 84-103. doi:10.1080/1068316x.2015.1111367

Haig, B. D. (2014). Investigating the psychological world: Scientific method in the behavioral sciences. Cambridge MA: MIT Press.

Hanson, R. K., \& Morton-Bourgon, K. E. (2009). The accuracy of recidivism risk assessments for sexual offenders: a meta-analysis of 118 prediction studies. Psychological assessment, 21(1), 1.

Hart, S. D., \& Logan, C. (2011). Formulation of violent risk using evidence-based assessments: The Structured Professional Judgement Approach. In P. Sturmey \& M. McMurran (Eds.), Forensic case formulation (pp. 3-32). West Sussex, UK: John Wiley \& Sons.

Hart, S. D., Sturmey, P., Logan, C., \& McMurran, M. (2011). Forensic case formulation. International Journal of Forensic Mental Health, 10, 118-126. DOI: 10.1080/14999013.2011.577137

Heffernan, R., \& Ward, T. (2015). The conceptualization of dynamic risk factors in child sex offenders: An agency model. Aggression and Violent Behavior,24, 250-260. doi.org/10.1016/j.avb.2015.07.001 
Heffernan, R., \& Ward, T. (2017). A comprehensive theory of dynamic risk and protective factors. Aggression and Violent Behavior, 37, 129-141. https://doi.org/10.1016/j.avb.2017.10.003

Heffernan, R., \& Ward, T. (in press). Dynamic risk factors, protective factors, and value-laden practices.

Lewis, G., \& Doyle, M. (2009). Risk formulation: What are we doing and why?. International Journal of Forensic Mental Health, 8(4), 286-292.

Lilienfeld, S. O. (2014). The Research Domain Criteria (RDoC): An analysis of methodological and conceptual challenges. Behaviour Research and Therapy, 62, 129-139. doi.org/10.1016/j.brat.2014.07.019

Logan, C. \& Johnstone, L. (2013). Managing clinical risk: A guide to effective practice. Oxford, UK: Routledge.

Mann, R. E., Hanson, R. K., \& Thornton, D. (2010). Assessing risk for sexual recidivism: some proposals on the nature of psychologically meaningful risk factors. Sex Abuse, 22(2), 191-217. https://doi.org/10.1177/1079063210366039

Murphy, D. (2006). Psychiatry in the scientific image. Cambridge, MA: MIT Press.

Olver, M. E., Stockdale, K. C., \& Wormith, J. S. (2014). Thirty years of research on the Level of Service Scales: A meta-analytic examination of predictive accuracy and sources of variability. Psychological Assessment, 26(1), 156.

Serin, R. C., Chadwick, N., \& Lloyd, C. D. (2016). Dynamic risk and protective factors. Psychology, Crime \& Law, 22(1-2), 151-170.

Smallbone, S. W., \& Dadds, M. R. (2000). Attachment and coercive sexual behavior. Sexual Abuse: A Journal of Research and Treatment, 12, 3-15.

Sturmey, P., \& McMurran, M. (Eds). (2011). Forensic case formulation. Oxford: Wiley-Blackwell. 
Thornton, D. (2016). Developing a theory of dynamic risk. Psychology, Crime \& Law, 22(1-2), 138-150.

Ward, T., \& Beech, A. R. (2015). Dynamic risk factors: A theoretical dead-end?

Psychology, Crime \& Law, 21, 100-113.

https://doi.org/10.1080/1068316x.2014.917854

Ward, T., Clack, S., \& Haig, B. D. (2016). The abductive theory of method: Scientific inquiry and clinical practice. Behaviour Change, 33(4), 212-231.

Ward, T., \& Fortune, C.-A. (2016a). The role of dynamic risk factors in the explanation of offending. Aggression and Violent Behavior, 29, 79-88. doi:10.1016/j.avb.2016.06.007

Ward, T., \& Fortune, C.A. (2016b). From dynamic risk factors to causal processes: A methodological framework. Psychology, Crime \& Law, 22, 190-202. https://doi.org/10.1080/1068316x.2015.1117080.

Yates, P. M., \& Kingston, D. A. (2006). The self-regulation model of sexual offending: The relationship between offence pathways and static and dynamic sexual offence risk. Sexual Abuse: A Journal of Research and Treatment, 18, 259-270. doi: 10.1007/s11194-006-9018-z 


\section{Table 1. Supported and Promising* Psychologically Meaningful Risk Factors (Mann et}

al., 2010)

\begin{tabular}{|c|c|c|c|}
\hline Domain & DRF & Types of practices & E.g., causal processes \\
\hline Sexual & $\begin{array}{l}\text { Sexual preoccupation } \\
\text { Any deviant sexual interest: } \\
\text { e.g., children, violence, multiple } \\
\text { paraphilia }\end{array}$ & $\begin{array}{l}\text { Sexual acts, seeking sex, } \\
\text { frequency, number/characteristics } \\
\text { of preferred partners }\end{array}$ & $\begin{array}{l}\text { Sexual drive, arousal, attraction } \\
\text { (preference), acceptance of sexual } \\
\text { identity, sexual scripts/schema }\end{array}$ \\
\hline Intimacy & $\begin{array}{l}\text { Emotional congruence with } \\
\text { children } \\
\text { Lack of emotionally intimate } \\
\text { relationships with adults: e.g., } \\
\text { never married, conflicts in } \\
\text { intimate relationships } \\
\text { Callousness/lack of concern for } \\
\text { others* }\end{array}$ & $\begin{array}{l}\text { Partner choice, communication, } \\
\text { establishing and maintaining } \\
\text { bonds, vulnerable disclosure, } \\
\text { caring for others, negotiating } \\
\text { conflict, commitment }\end{array}$ & $\begin{array}{l}\text { Interpersonal skills, preferences } \\
\text { for emotional intimacy, capacity } \\
\text { for sexual and emotional } \\
\text { connection, attachment processes, } \\
\text { language and communication }\end{array}$ \\
\hline $\begin{array}{l}\text { Self- } \\
\text { regulation }\end{array}$ & $\begin{array}{l}\text { Lifestyle impulsivity } \\
\text { General self-regulation } \\
\text { problems: e.g., impulsivity, } \\
\text { recklessness, employment } \\
\text { instability } \\
\text { Poor cognitive problem-solving } \\
\text { Resistance to rules/supervision: } \\
\text { e.g., childhood behavioral } \\
\text { problems, non-compliance with } \\
\text { supervision, violation of } \\
\text { conditional release } \\
\text { Dysfunctional coping: e.g., } \\
\text { sexualized, externalizing* }\end{array}$ & $\begin{array}{l}\text { Seeking employment, managing } \\
\text { finances, leisure activities, self- } \\
\text { care, compliance with rules/law, } \\
\text { problem solving, planning and } \\
\text { goal setting, communication of } \\
\text { emotions, coping }\end{array}$ & $\begin{array}{l}\text { Motivation, self-control, skills } \\
\text { relevant to context (e.g., attitudes, } \\
\text { conflict resolution), emotion: } \\
\text { recognition, interpretation, control }\end{array}$ \\
\hline Attitudes & $\begin{array}{l}\text { Offense-supportive attitudes } \\
\text { Hostility toward women* } \\
\text { Machiavellianism* }\end{array}$ & $\begin{array}{l}\text { Representing reality, causal } \\
\text { reasoning, } \\
\text { interpretation/attribution, } \\
\text { explaining and justifying action }\end{array}$ & $\begin{array}{l}\text { Memory, causal reasoning, theory } \\
\text { of mind, cognitive flexibility, } \\
\text { interpreting input, } \\
\text { accuracy (i.e., based on evidence) }\end{array}$ \\
\hline
\end{tabular}




\begin{tabular}{|c|c|c|}
\hline Phase 1: Deconstruction & Phase 2: Analysis & Phase 3: Reintegration \\
\hline $\begin{array}{l}\text { Identify candidate DRF, } \\
\text { exemplar, breakdown into: }\end{array}$ & $\begin{array}{l}\text { Analyse causal processes, } \\
\text { acquire evidence across } \\
\text { DRRF levels: }\end{array}$ & $\begin{array}{l}\text { Reintegrate local theories } \\
\text { within agency model: }\end{array}$ \\
\hline 1. Symptoms & 1 Piglonical & Illustrates the role of each \\
\hline $\begin{array}{l}\text { 3. Possible causal } \\
\text { strands (i.e., DRRF } \\
\text { causal processes) }\end{array}$ & $\begin{array}{l}\text { 1. Piological } \\
\text { 3. Behomenological } \\
\text { 4. Contextual }\end{array}$ & $\begin{array}{l}\text { causal process in norm- } \\
\text { violation, integrates these } \\
\text { impairments with normative } \\
\text { practices and contexts. }\end{array}$ \\
\hline $\begin{array}{l}\text { Output: possible causal } \\
\text { processes, multiple for each } \\
\text { candidate DRF }\end{array}$ & $\begin{array}{l}\text { Output: multi-level local } \\
\text { theories for each causal } \\
\text { process }\end{array}$ & $\begin{array}{l}\text { Output: a model of } \\
\text { behaviour with local } \\
\text { theories integrated within }\end{array}$ \\
\hline
\end{tabular}

Figure 1. Risk-Causality Method of Deconstruction, Analysis, and Reintegration.

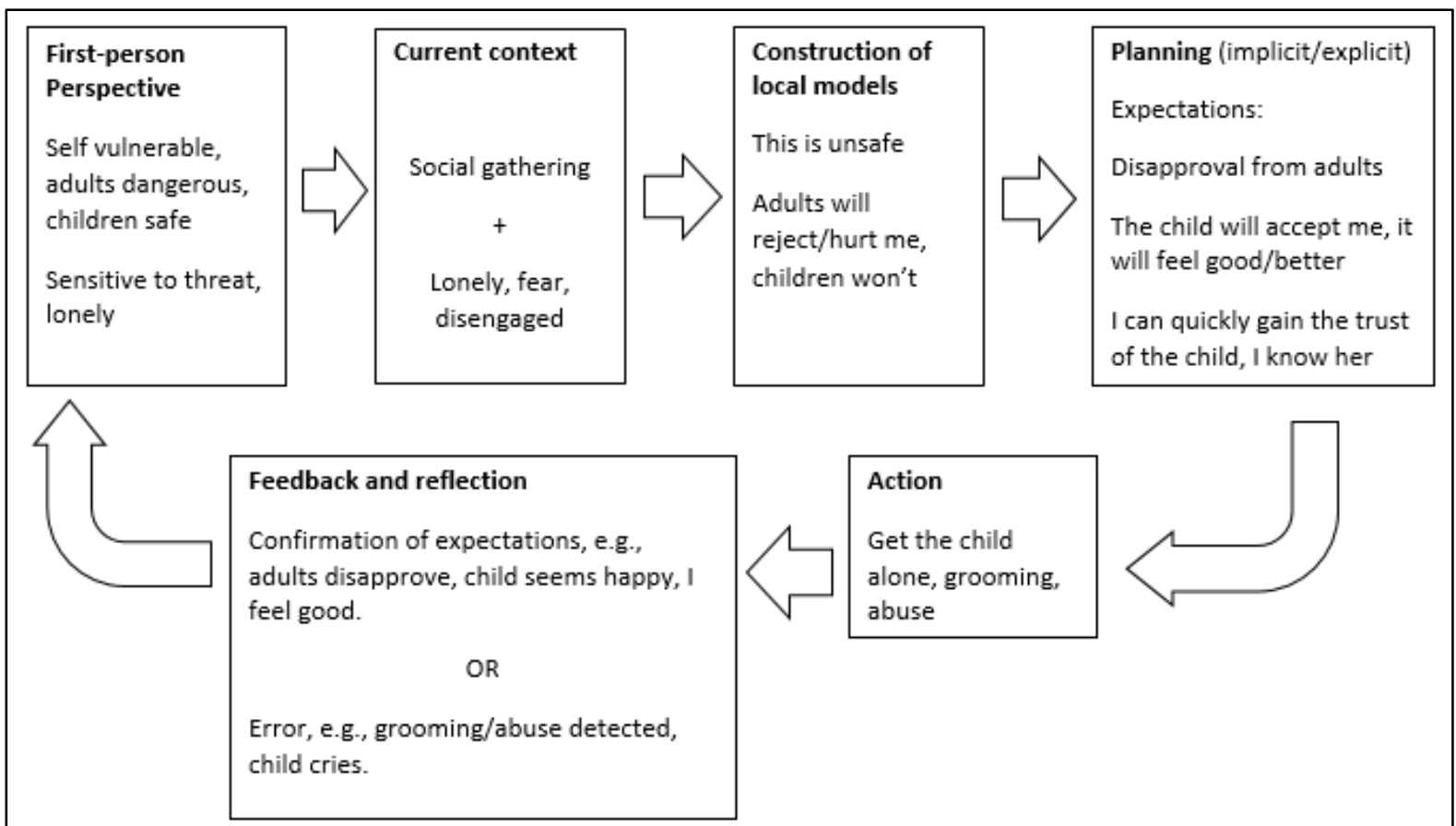

Figure 2. Predictive Agency Model: threat detection and emotional congruence with children. 